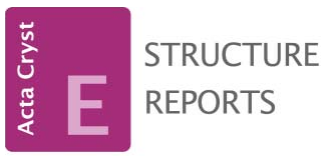

ISSN 1600-5368

Received 7 November 2014

Accepted 24 November 2014

Edited by W. T. A. Harrison, University of Aberdeen, Scotland

Keywords: crystal structure; $\beta$-bromodehydroalanine; dehydroamino acid; non-helical conformation; hydrogen bonding

CCDC reference: 1035539

Supporting information: this article has supporting information at journals.iucr.org/e

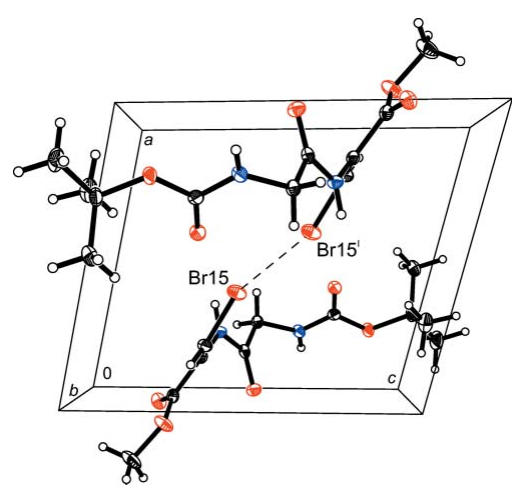

\section{Crystal structure of $\mathrm{N}$-(tert-butoxycarbonyl)glycyl- $(Z)$ - $\beta$-bromodehydroalanine methyl ester [Boc-Gly- $\left.(\beta-\mathrm{Br})^{(\mathrm{Z})} \Delta \mathrm{Ala}-\mathrm{OMe}\right]$}

\author{
Paweł Lenartowicz, Maciej Makowski, Bartosz Zarychta* and Krzysztof Ejsmont
}

Faculty of Chemistry, University of Opole, Oleska 48, 45-052 Opole, Poland. *Correspondence e-mail:

bzarychta@uni.opole.pl

The title compound, $\mathrm{C}_{11} \mathrm{H}_{17} \mathrm{BrN}_{2} \mathrm{O}_{5}$, is a dehydroamino acid with a $\mathrm{C}=\mathrm{C}$ bond between the $\alpha$ - and $\beta$-C atoms. The amino acid residues are linked trans to each other and there are no strong intramolecular hydrogen bonds. The torsion angles indicate a non-helical conformation of the molecule. The dipeptide folding is influenced by an intermolecular $\mathrm{N}-\mathrm{H} \cdots \mathrm{O}$ hydrogen bond and also minimizes steric repulsion. In the crystal, molecules are linked by strong $\mathrm{N}-$ $\mathrm{H}$. . O hydrogen bonds, generating (001) sheets. The sheets are linked by weak $\mathrm{C}-\mathrm{H} \cdots \mathrm{O}$ and $\mathrm{C}-\mathrm{H} \cdots \mathrm{Br}$ bonds and short $\mathrm{Br} \cdots \mathrm{Br}[3.4149$ (3) $\AA$ ] interactions.

\section{Chemical context}

Dehydroamino acids are analogues of amino acids characterized by the presence of an unsaturated doubled bound between the $\alpha$ - and $\beta$-carbon atoms in their structure. These compounds were found to be components of natural products (Bonauer et al., 2006), with lantibiotics being especially important since they are an important class of natural bacteriocins produced by Gram-positive bacteria (Willey \& van der Donk, 2007). The development of synthetic methods for the preparation of dehydropeptides allows researchers to search for their practical applications and to use them as substrates for the production of peptidomimetics. One of the interesting classes of such mimetics are $\beta$-bromo-dehydroamino acids and their derivatives, which are usually obtained by radical halogenation of dehydroamino acids using $N$ bromosuccinimide (NBS). This reaction proceeds in two steps, namely by halogenation of dehydroamino acids, which gives $\alpha$-bromo-imines, followed by tautomerization to the desired products upon treatment with an amine (Coleman \& Carpenter, 1993; Zhang et al., 2002). $\beta$-Bromo-dehydroamino acid derivatives are useful substrates in coupling reactions with alkynes (Singh et al., 2003) or organoboranes (Collier et al. 2002; Zhang et al., 2002). Further asymmetric hydrogenation of their double bound allows non-proteinogenic $\alpha$-amino acids and their derivatives to be obtained. Another important reaction of $\beta$-bromo- $\alpha, \beta$-dehydroamino acid derivatives in drug research is their coupling cyclization in which oxazole derivatives are produced (Liu et al., 2014).<smiles>COC(=O)/C(=C\Br)NC(=O)CNC(=O)OC(C)(C)C</smiles>

\title{
OPEN $\odot$ ACCESS
}




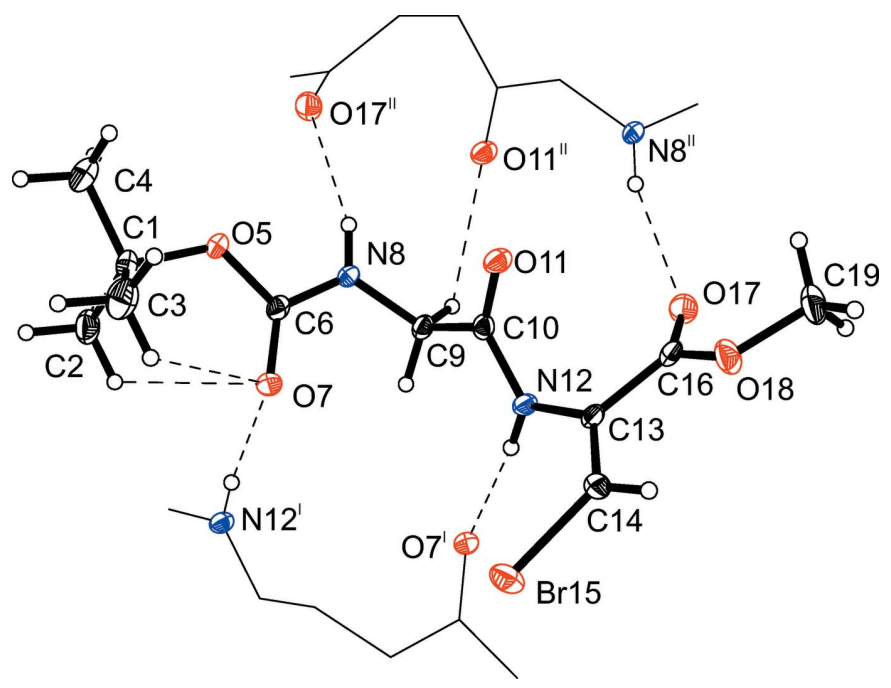

Figure 1

The molecular structure of Boc-Gly- $(\beta-\mathrm{Br})^{(Z)} \Delta \mathrm{Ala}-\mathrm{OMe}$ along with selected intramolecular hydrogen bonds (dashed lines), drawn with $50 \%$ displacement ellipsoids.

\section{Structural commentary}

The molecular structure of the title compound, (I), is shown in Fig. 1. The amino acids in the compound are linked trans to each other. The $\omega^{2}$ angle $(\mathrm{C} 9-\mathrm{C} 10-\mathrm{N} 12-\mathrm{C} 13)$ is $175.79(16)^{\circ}$, while $\omega^{3}(\mathrm{O} 5-\mathrm{C} 6-\mathrm{N} 8-\mathrm{C} 9)$ is $176.12(15)^{\circ}$. There are no strong intramolecular hydrogen bonds. The values of the $\varphi^{2,3}$ and $\psi^{2,3}$ angles corresponds to a non-helical conformation (Venkatachalam, 1968). The dipeptide folds accordingly to the intermolecular $\mathrm{N}-\mathrm{H}$... O-type hydrogen bonds. The $\beta$-bromo-dehydroalanine moiety shows typical geometrical tendencies. The $\mathrm{C} 10-\mathrm{N} 12$ bond is longer [1.366 (2) $\AA]$ than a typical bond in alanine, while the N12C13 bond is shorter [1.406 (2) $\AA$ ]. This effect is common for other dehydro-residues (Ajó et al., 1979; Pieroni et al. 1975; Rzeszotarska et al., 2002; Jain \& Chauhan, 1996). This indicates conjugation between the side chain of dehydroalanine and the peptide bond. The torsion angles around the $\mathrm{Br}(\mathrm{H}) \mathrm{C}=\mathrm{C}$ grouping are $-0.9(3)$ and $-174.28(13)^{\circ}(\mathrm{N} 12-$ $\mathrm{C} 13-\mathrm{C} 14-\mathrm{Br} 15$ and $\mathrm{C} 16-\mathrm{C} 13-\mathrm{C} 14-\mathrm{Br} 15$, respectively), meaning the stereochemistry about the bond is especially planar. This is consistent with the nature of an $s p^{2}$-hybridized carbon on $\mathrm{C} 13$. The valance angles around the dehydroalanine group show some unusual values, especially $\mathrm{N} 12-\mathrm{C} 13-\mathrm{C} 14$ [124.27 $\left.(18)^{\circ}\right]$, which may correspond to the presence of the bromine atom in the structure. The other angles are normal, as the backbone of the molecule is folded to minimize steric repulsion. The Boc group features two short intramolecular $\mathrm{C}-\mathrm{H} \cdots \mathrm{O}$ contacts

\section{Supramolecular features}

In the crystal, molecules form two strong twin $\mathrm{N}-\mathrm{H} \cdots \mathrm{O}$ $\left(\mathrm{N} 8-\mathrm{H} 8 A \cdots \mathrm{O} 17^{\mathrm{i}}\right.$ and $\left.\mathrm{N} 12-\mathrm{H} 12 A \cdots \mathrm{O} 7^{\mathrm{ii}}\right)$ and one weak accompanying $\mathrm{C} 9-\mathrm{H} 9 A \cdots \mathrm{O} 11^{\mathrm{i}}$ hydrogen bonds (Fig. 1 and
Table 1

Hydrogen-bond geometry $\left(\AA,{ }^{\circ}\right)$.

\begin{tabular}{|c|c|c|c|c|}
\hline$D-\mathrm{H} \cdots A$ & $D-\mathrm{H}$ & $\mathrm{H} \cdots A$ & $D \cdots A$ & $D-\mathrm{H} \cdots A$ \\
\hline $\mathrm{C} 2-\mathrm{H} 2 A \cdots \mathrm{O} 7$ & 0.96 & 2.51 & $3.058(2)$ & 116 \\
\hline $\mathrm{C} 3-\mathrm{H} 3 A \cdots \mathrm{O} 7$ & 0.96 & 2.44 & $3.007(3)$ & 117 \\
\hline $\mathrm{N} 8-\mathrm{H} 8 A \cdots \mathrm{O} 17^{\mathrm{i}}$ & 0.86 & 2.19 & $3.018(2)$ & 162 \\
\hline $\mathrm{C} 9-\mathrm{H} 9 A \cdots \mathrm{O} 11^{\mathrm{i}}$ & 0.97 & 2.61 & $3.255(2)$ & 124 \\
\hline $\mathrm{N} 12-\mathrm{H} 12 A \cdots \mathrm{O} 7^{\mathrm{ii}}$ & 0.86 & 2.04 & $2.901(2)$ & 174 \\
\hline $\mathrm{C} 14-\mathrm{H} 14 A \cdots \mathrm{O} 11^{\mathrm{iii}}$ & 0.93 & 2.43 & $3.095(2)$ & 129 \\
\hline $\mathrm{C} 19-\mathrm{H} 19 B \cdots \mathrm{Br} 15^{\mathrm{iii}}$ & 0.96 & 3.14 & $3.668(3)$ & 117 \\
\hline
\end{tabular}

Table 1), forming infinite sheets in the (001) plane [symmetry codes: (i) $-x+2,-y,-z+1$ and (ii) $-x+3,-y,-z+1]$. The sheets are connected to each other by weak $\mathrm{C} 14-$ $\mathrm{H} 14 A \cdots \mathrm{O} 11^{\mathrm{iii}}$ and $\mathrm{C} 19-\mathrm{H} 19 B \cdots \mathrm{Br} 15^{\mathrm{iii}}$ hydrogen bonds and one $\mathrm{Br} \cdots \mathrm{Br}^{\mathrm{iv}}[3.4149$ (3) $\AA$ ] halogen bond (Fig. 2) of type I (Mukherjee \& Desiraju, 2014) [symmetry codes: (iii) $-x+2$, $-y+1,-z+1$; (iv) $-x+3,-y+1,-z+1]$.

\section{Synthesis and crystallization}

Boc-Gly- $\Delta$ Ala and its methyl ester were prepared according to the methodology described by Makowski et al. (1985) and Cossec et al. (2008). The $\beta$-bromo-vinyl derivative was obtained based on a procedure described previously (Bull et al., 2007). For this purpose $0.129 \mathrm{~g}(0.5 \mathrm{mM})$ of Boc-Gly$\triangle \mathrm{Ala}-\mathrm{OMe}$ was dissolved in $2.5 \mathrm{ml}$ of dichloromethane and cooled to $193 \mathrm{~K}$. Then, bromine $0.027 \mathrm{ml}(0.5 \mathrm{mM})$ was added. The solution was stirred over 10 minutes followed by addition of triethylamine $0.210 \mathrm{ml}(1.5 \mathrm{mM})$. After 15 minutes, the mixture was quenched with $20 \mathrm{ml}$ of saturated aqueous

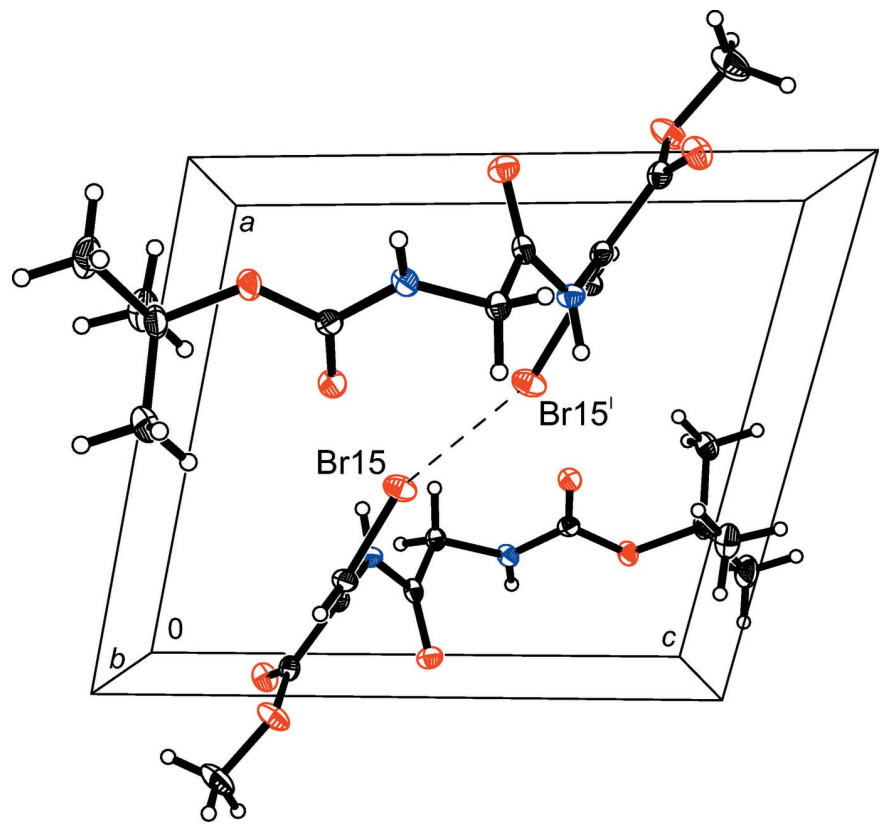

Figure 2

A packing diagram of (I), viewed along the $b$ axis, showing the intermolecular hydrogen-bonding scheme (dashed lines). 
$\mathrm{NaHCO}_{3}$ and warmed to room temperature. The product was extracted by dichloromethane $(3 \times 15 \mathrm{ml})$. The organic layer was washed with brine $(3 \times 10 \mathrm{ml})$ and dried over anhydrous $\mathrm{Na}_{2} \mathrm{SO}_{4}$. Evaporation of the solvent at reduced pressure gave $0.119 \mathrm{~g}(0.35 \mathrm{mM})$ of crude product $(70 \%$ yield). Recrystallization was performed from mixtures of diethyl ether/ethyl acetate(2:1)/hexane solvents, yielding irregular colourless crystals. It is worth noting that in the case of our study, the formation of only the $Z$ isomer was observed while in the preceding paper, the bromination of dehydroalaninecontaining compound gave the $E$ isomer. ${ }^{1} \mathrm{H}$ NMR $(400 \mathrm{MHz}$, DMSO) $\delta 1.38\left(s,\left(s, 9 \mathrm{H}, \mathrm{C}-\mathrm{H}_{3} t\right.\right.$-Boc $), 3.67\left(s, 3 \mathrm{H}, \mathrm{O}-\mathrm{CH}_{3}\right)$, $3.69\left(d, J=6.2 \mathrm{~Hz}, 2 \mathrm{H}, \mathrm{C}-\mathrm{H}_{2}\right.$ Gly $), 7.05(t, J=6.2 \mathrm{~Hz}, 1 \mathrm{H}, \mathrm{N}-$ $\left.\mathrm{H}_{\mathrm{Gly}}\right), 7.30(s, 1 \mathrm{H}, \mathrm{C}=\mathrm{CHBr}), 9.63\left(\mathrm{~s}, 1 \mathrm{H}, \mathrm{N}-\mathrm{H}_{\beta-\mathrm{Br}-\Delta \mathrm{Ala}}\right)$. ${ }^{13} \mathrm{C}$ NMR (101 MHz, DMSO) $\delta$ 28.21, 42.79, 52.54, 78.12, $113.26,132.88$, 155.80, 162.63, 168.80. Melting point $=386-$ $388 \mathrm{~K}$.

\section{Refinement}

Crystal data, data collection and structure refinement details are summarized in Table 2. All $\mathrm{H}$ atoms were positioned geometrically and treated as riding on their parent $\mathrm{C}$ or $\mathrm{N}$ atoms: for methyl groups, $\mathrm{C}-\mathrm{H}=0.96 \AA$ and $U_{\text {iso }}(\mathrm{H})=$ $1.5 U_{\mathrm{eq}}(\mathrm{C})$; for $\mathrm{N}$ atoms, $\mathrm{N}-\mathrm{H}=0.86 \AA$ and $U_{\text {iso }}(\mathrm{H})=$ $1.2 U_{\text {eq }}(\mathrm{C})$; for secondary $\mathrm{C}$ atoms, $\mathrm{C}-\mathrm{H}=0.97 \AA$ and $U_{\text {iso }}(\mathrm{H})$ $=1.2 U_{\mathrm{eq}}(\mathrm{C})$, with no refinement of their parameters.

\section{Acknowledgements}

This study was supported by the Wroclaw Research Centre EIT+ under the project Biotechnologies and advanced medical technologies - BioMed (POIG.01.01.02-02-003/08) financed from the European Regional Development Fund (Operational Programme Innovative Economy, 1.1.2). PL is the recipient of a PhD fellowships from a project funded by the European Social Fund.

\section{References}

Ajó, D., Granozzi, G., Tondello, E., Del Pra, A. \& Zanotti, G. (1979). J. Chem. Soc. Perkin Trans. 2, pp. 927-929.

Bonauer, C., Walenzyk, T. \& König, B. (2006). Synthesis, pp. 1-20.

Bull, J. A., Balskus, E. P., Horan, R. A. J., Langner, M. \& Ley, S. V. (2007). Chem. Eur. J. 13, 5515-5538.

Coleman, R. S. \& Carpenter, A. J. (1993). J. Org. Chem. 58, 44524461.

Collier, P. N., Campbell, A. D., Patel, I., Raynham, T. M. \& Taylor, R. J. K. (2002). J. Org. Chem. 67, 1802-1815.

Cossec, B., Cosnier, F. \& Burgart, M. (2008). Molecules, 13, 2394 2407.
Table 2

Experimental details.

\begin{tabular}{|c|c|}
\hline \multicolumn{2}{|l|}{ Crystal data } \\
\hline Chemical formula & $\mathrm{C}_{11} \mathrm{H}_{17} \mathrm{BrN}_{2} \mathrm{O}_{5}$ \\
\hline$M_{\mathrm{r}}$ & 337.17 \\
\hline Crystal system, space group & Triclinic, $P \overline{1}$ \\
\hline Temperature $(\mathrm{K})$ & 100 \\
\hline$a, b, c(\AA)$ & $9.0431(4), 9.3160(4), 9.7540$ (4) \\
\hline$\alpha, \beta, \gamma\left({ }^{\circ}\right)$ & $83.381(3), 75.420(4), 64.863(4)$ \\
\hline$V\left(\AA^{3}\right)$ & $719.92(6)$ \\
\hline$Z$ & 2 \\
\hline Radiation type & Мo $K \alpha$ \\
\hline$\mu\left(\mathrm{mm}^{-1}\right)$ & 2.87 \\
\hline Crystal size $(\mathrm{mm})$ & $0.30 \times 0.25 \times 0.20$ \\
\hline \multicolumn{2}{|l|}{ Data collection } \\
\hline Diffractometer & Oxford Diffraction Xcalibur \\
\hline Absorption correction & $\begin{array}{l}\text { Multi-scan (CrysAlis RED; Oxford } \\
\text { Diffraction, 2010) }\end{array}$ \\
\hline$T_{\min }, T_{\max }$ & $0.655,1.000$ \\
\hline $\begin{array}{l}\text { No. of measured, independent and } \\
\text { observed }[I>2 \sigma(I)] \text { reflections }\end{array}$ & $4860,2780,2490$ \\
\hline$R_{\text {int }}$ & 0.016 \\
\hline$(\sin \theta / \lambda)_{\max }\left(\AA^{-1}\right)$ & 0.617 \\
\hline \multicolumn{2}{|l|}{ Refinement } \\
\hline$R\left[F^{2}>2 \sigma\left(F^{2}\right)\right], w R\left(F^{2}\right), S$ & $0.024,0.066,1.06$ \\
\hline No. of reflections & 2780 \\
\hline No. of parameters & 172 \\
\hline $\mathrm{H}$-atom treatment & H-atom parameters constrained \\
\hline$\Delta \rho_{\max }, \Delta \rho_{\min }\left(\mathrm{e} \AA^{-3}\right)$ & $0.53,-0.43$ \\
\hline
\end{tabular}

Computer programs: CrysAlis CCD and CrysAlis RED (Oxford Diffraction, 2010), SHELXS2014 and SHELXL2014 (Sheldrick, 2008), SHELXTL (Sheldrick, 2008).

Jain, R. \& Chauhan, V. S. (1996). Biopolymers, 40, 105-119.

Liu, B., Zhang, Y., Huang, G., Zhang, X., Niu, P., Wu, J., Yu, W. \& Chang, J. (2014). Org. Biomol. Chem. 12, 3912-3923.

Makowski, M., Rzeszotarska, B., Kubica, R. \& Pietrzyński, G. (1985). Liebigs Ann. Chem. 5, 893-900.

Mukherjee, A. \& Desiraju, G. R. (2014). IUCrJ, 1, 49-60.

Oxford Diffraction (2010). CrysAlisCCD and CrysAlisRED. Oxford Diffraction Ltd, Abingdon, England.

Pieroni, O., Montagnoli, G., Fissi, A., Merlino, S. \& Ciardelli, F. (1975). J. Am. Chem. Soc. 1, 6820-6826.

Rzeszotarska, B., Siodłak, D., Broda, M. A., Dybała, I. \& Kozioł, A. E. (2002). J. Pept. Res. 59, 79-89.

Sheldrick, G. M. (2008). Acta Cryst. A64, 112-122.

Singh, J., Kronenthal, D. R., Schwinden, M., Godfrey, J. D., Fox, R., Vawter, E. J., Zhang, B., Kissick, T. P., Patel, B., Mneimne, O., Humora, M., Papaioannou, C. G., Szymanski, W., Wong, M. K. Y., Chen, C. K., Heikes, J. E., DiMarco, J. D., Qiu, J., Deshpande, R. P., Gougoutas, J. Z. \& Mueller, R. H. (2003). Org. Lett. 5, 3155-3158. Venkatachalam, C. M. (1968). Biopolymers, 6, 1425-1436.

Willey, J. M. \& van der Donk, W. A. (2007). Annu. Rev. Microbiol. 61, 477-501.

Zhang, J. Y., Xiong, C. Y., Wang, W., Ying, J. F. \& Hruby, V. J. (2002). Org. Lett. 4, 4029-4032. 


\section{supporting information}

Acta Cryst. (2014). E70, 596-598 [doi:10.1107/S1600536814025677]

\section{Crystal structure of $N$-(tert-butoxycarbonyl)glycyl-(Z)- $\beta$-bromodehydroalanine methyl ester [Boc-Gly- $(\beta \text {-Br })^{(Z)} \Delta$ Ala-OMe]}

\section{Pawel Lenartowicz, Maciej Makowski, Bartosz Zarychta and Krzysztof Ejsmont}

\section{Computing details}

Data collection: CrysAlis CCD (Oxford Diffraction, 2010); cell refinement: CrysAlis RED (Oxford Diffraction, 2010); data reduction: CrysAlis RED (Oxford Diffraction, 2010); program(s) used to solve structure: SHELXS2014 (Sheldrick, 2008); program(s) used to refine structure: SHELXL2014 (Sheldrick, 2008); molecular graphics: SHELXTL (Sheldrick, 2008); software used to prepare material for publication: SHELXL2014 (Sheldrick, 2008).

\section{$N$-(tert-Butoxycarbonyl)glycyl-(Z)- $\beta$-bromodehydroalanine methyl ester}

\section{Crystal data}

$\mathrm{C}_{11} \mathrm{H}_{17} \mathrm{BrN}_{2} \mathrm{O}_{5}$

$M_{r}=337.17$

Triclinic, $P \overline{1}$

$a=9.0431(4) \AA$

$b=9.3160(4) \AA$

$c=9.7540(4) \AA$

$\alpha=83.381(3)^{\circ}$

$\beta=75.420(4)^{\circ}$

$\gamma=64.863(4)^{\circ}$

$V=719.92(6) \AA^{3}$

\section{Data collection}

Oxford Diffraction Xcalibur diffractometer

Radiation source: fine-focus sealed tube Graphite monochromator

Detector resolution: 1024 pixels $\mathrm{mm}^{-1}$

$\omega$ scan

Absorption correction: multi-scan

(CrysAlis RED; Oxford Diffraction, 2010)

$T_{\min }=0.655, T_{\max }=1.000$

\section{Refinement}

Refinement on $F^{2}$

Least-squares matrix: full

$R\left[F^{2}>2 \sigma\left(F^{2}\right)\right]=0.024$

$w R\left(F^{2}\right)=0.066$

$S=1.06$

2780 reflections

172 parameters

0 restraints

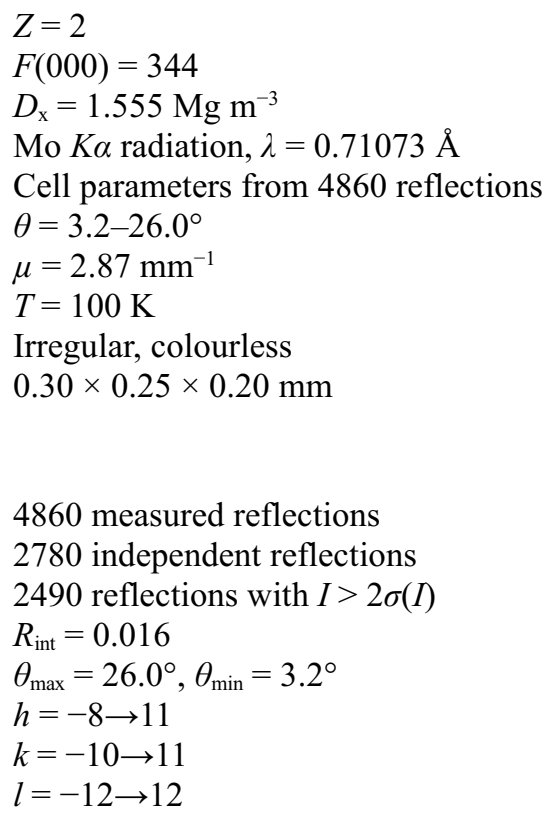

4860 measured reflections 2780 independent reflections 2490 reflections with $I>2 \sigma(I)$

$R_{\text {int }}=0.016$

$\theta_{\text {max }}=26.0^{\circ}, \theta_{\text {min }}=3.2^{\circ}$

$h=-8 \rightarrow 11$

$k=-10 \rightarrow 11$

$l=-12 \rightarrow 12$

Hydrogen site location: inferred from neighbouring sites

$\mathrm{H}$-atom parameters constrained

$w=1 /\left[\sigma^{2}\left(F_{\mathrm{o}}{ }^{2}\right)+(0.0444 P)^{2}\right]$

where $P=\left(F_{\mathrm{o}}{ }^{2}+2 F_{\mathrm{c}}{ }^{2}\right) / 3$

$(\Delta / \sigma)_{\max }=0.001$

$\Delta \rho_{\max }=0.53$ e $\AA^{-3}$

$\Delta \rho_{\min }=-0.43$ e $\AA^{-3}$ 
Special details

Experimental. CrysAlis RED, Oxford Diffraction Ltd., Version 1.171.33.57 (release 26-01-2010 CrysAlis171 .NET) (compiled Jan 26 2010,14:36:55) Empirical absorption correction using spherical harmonics, implemented in SCALE3 ABSPACK scaling algorithm.

Geometry. All e.s.d.'s (except the e.s.d. in the dihedral angle between two 1.s. planes) are estimated using the full covariance matrix. The cell e.s.d.'s are taken into account individually in the estimation of e.s.d.'s in distances, angles and torsion angles; correlations between e.s.d.'s in cell parameters are only used when they are defined by crystal symmetry. An approximate (isotropic) treatment of cell e.s.d.'s is used for estimating e.s.d.'s involving 1.s. planes.

Fractional atomic coordinates and isotropic or equivalent isotropic displacement parameters $\left(\AA^{2}\right)$

\begin{tabular}{|c|c|c|c|c|}
\hline & $x$ & $y$ & $z$ & $U_{\text {iso }} * / U_{\text {eq }}$ \\
\hline $\mathrm{C} 1$ & $1.2957(3)$ & $-0.1495(2)$ & $0.9892(2)$ & $0.0186(4)$ \\
\hline $\mathrm{C} 2$ & $1.4783(3)$ & $-0.2662(3)$ & $0.9655(2)$ & $0.0248(5)$ \\
\hline $\mathrm{H} 2 \mathrm{~A}$ & 1.5455 & -0.2243 & 0.8952 & $0.037^{*}$ \\
\hline $\mathrm{H} 2 \mathrm{~B}$ & 1.4904 & -0.3651 & 0.9336 & $0.037 *$ \\
\hline $\mathrm{H} 2 \mathrm{C}$ & 1.5146 & -0.2830 & 1.0527 & $0.037^{*}$ \\
\hline $\mathrm{C} 3$ & $1.2675(3)$ & $0.0136(3)$ & $1.0284(2)$ & $0.0291(5)$ \\
\hline $\mathrm{H} 3 \mathrm{~A}$ & 1.3374 & 0.0518 & 0.9572 & $0.044 *$ \\
\hline H3B & 1.2952 & 0.0083 & 1.1182 & $0.044 *$ \\
\hline $\mathrm{H} 3 \mathrm{C}$ & 1.1520 & 0.0844 & 1.0348 & $0.044^{*}$ \\
\hline $\mathrm{C} 4$ & $1.1870(3)$ & $-0.2140(3)$ & $1.1000(2)$ & $0.0313(5)$ \\
\hline $\mathrm{H} 4 \mathrm{~A}$ & 1.0720 & -0.1387 & 1.1146 & $0.047 *$ \\
\hline H4B & 1.2228 & -0.2316 & 1.1875 & $0.047 *$ \\
\hline $\mathrm{H} 4 \mathrm{C}$ & 1.1975 & -0.3122 & 1.0679 & $0.047^{*}$ \\
\hline O5 & $1.23184(17)$ & $-0.14194(16)$ & $0.86239(14)$ & $0.0186(3)$ \\
\hline C6 & $1.2959(2)$ & $-0.0915(2)$ & $0.7363(2)$ & $0.0144(4)$ \\
\hline O7 & $1.40332(16)$ & $-0.03851(15)$ & $0.71486(14)$ & $0.0161(3)$ \\
\hline N8 & $1.2267(2)$ & $-0.10813(19)$ & $0.63638(17)$ & $0.0155(3)$ \\
\hline $\mathrm{H} 8 \mathrm{~A}$ & 1.1573 & -0.1522 & 0.6578 & $0.019 *$ \\
\hline C9 & $1.2670(2)$ & $-0.0531(2)$ & 0.49289 (19) & $0.0149(4)$ \\
\hline H9A & 1.2529 & -0.1157 & 0.4283 & $0.018^{*}$ \\
\hline H9B & 1.3835 & -0.0685 & 0.4688 & $0.018 *$ \\
\hline $\mathrm{C} 10$ & $1.1567(2)$ & $0.1209(2)$ & $0.47430(19)$ & $0.0143(4)$ \\
\hline O11 & $1.00831(16)$ & $0.18140(15)$ & $0.53245(14)$ & $0.0179(3)$ \\
\hline N12 & $1.23628(19)$ & $0.20273(18)$ & $0.38410(17)$ & $0.0146(3)$ \\
\hline $\mathrm{H} 12 \mathrm{~A}$ & 1.3421 & 0.1556 & 0.3484 & $0.017^{*}$ \\
\hline C13 & $1.1490(2)$ & $0.3623(2)$ & $0.34831(19)$ & $0.0136(4)$ \\
\hline C14 & $1.1990(2)$ & $0.4770(2)$ & $0.3536(2)$ & 0.0164 (4) \\
\hline $\mathrm{H} 14 \mathrm{~A}$ & 1.1341 & 0.5792 & 0.3266 & $0.020^{*}$ \\
\hline Br15 & $1.39326(2)$ & $0.44202(2)$ & $0.41252(2)$ & $0.02292(9)$ \\
\hline $\mathrm{C} 16$ & $1.0010(2)$ & $0.4006(2)$ & $0.2866(2)$ & $0.0163(4)$ \\
\hline O17 & $0.97138(18)$ & $0.30286(17)$ & $0.24167(15)$ & $0.0210(3)$ \\
\hline $\mathrm{O} 18$ & $0.90913(18)$ & $0.55685(16)$ & $0.28281(16)$ & $0.0239(3)$ \\
\hline C19 & $0.7741(3)$ & $0.6065(3)$ & 0.2098 (3) & $0.0324(5)$ \\
\hline H19A & 0.7155 & 0.7199 & 0.2129 & $0.049 *$ \\
\hline H19B & 0.6979 & 0.5594 & 0.2552 & $0.049^{*}$ \\
\hline H19C & 0.8193 & 0.5731 & 0.1130 & $0.049^{*}$ \\
\hline
\end{tabular}


Atomic displacement parameters $\left(\AA^{2}\right)$

\begin{tabular}{lllllll}
\hline & $U^{11}$ & $U^{22}$ & $U^{33}$ & $U^{12}$ & $U^{13}$ & $U^{23}$ \\
\hline C1 & $0.0226(10)$ & $0.0227(10)$ & $0.0121(9)$ & $-0.0104(9)$ & $-0.0065(8)$ & $0.0034(8)$ \\
C2 & $0.0266(11)$ & $0.0256(11)$ & $0.0202(11)$ & $-0.0075(9)$ & $-0.0108(9)$ & $0.0066(9)$ \\
C3 & $0.0397(14)$ & $0.0268(12)$ & $0.0204(11)$ & $-0.0129(10)$ & $-0.0070(10)$ & $-0.0014(9)$ \\
C4 & $0.0369(13)$ & $0.0432(14)$ & $0.0172(11)$ & $-0.0222(11)$ & $-0.0058(9)$ & $0.0088(10)$ \\
O5 & $0.0202(7)$ & $0.0248(7)$ & $0.0141(7)$ & $-0.0133(6)$ & $-0.0061(6)$ & $0.0077(6)$ \\
C6 & $0.0131(9)$ & $0.0102(9)$ & $0.0158(10)$ & $-0.0021(7)$ & $-0.0029(7)$ & $0.0040(7)$ \\
O7 & $0.0173(7)$ & $0.0166(7)$ & $0.0174(7)$ & $-0.0100(6)$ & $-0.0049(5)$ & $0.0028(5)$ \\
N8 & $0.0169(8)$ & $0.0164(8)$ & $0.0167(8)$ & $-0.0105(7)$ & $-0.0063(7)$ & $0.0074(7)$ \\
C9 & $0.0175(9)$ & $0.0136(9)$ & $0.0136(9)$ & $-0.0069(8)$ & $-0.0042(7)$ & $0.0038(7)$ \\
C10 & $0.0187(10)$ & $0.0152(9)$ & $0.0126(9)$ & $-0.0091(8)$ & $-0.0065(8)$ & $0.0017(7)$ \\
O11 & $0.0153(7)$ & $0.0149(7)$ & $0.0198(7)$ & $-0.0054(6)$ & $-0.0009(6)$ & $0.0035(5)$ \\
N12 & $0.0120(8)$ & $0.0130(8)$ & $0.0169(8)$ & $-0.0050(6)$ & $-0.0019(6)$ & $0.0034(6)$ \\
C13 & $0.0147(9)$ & $0.0143(9)$ & $0.0115(9)$ & $-0.0069(8)$ & $-0.0019(7)$ & $0.0031(7)$ \\
C14 & $0.0137(9)$ & $0.0164(9)$ & $0.0191(10)$ & $-0.0061(8)$ & $-0.0049(8)$ & $0.0021(8)$ \\
B15 & $0.01942(12)$ & $0.02164(12)$ & $0.03230(14)$ & $-0.01022(9)$ & $-0.00996(9)$ & $-0.00138(8)$ \\
C16 & $0.0181(10)$ & $0.0171(10)$ & $0.0130(9)$ & $-0.0083(8)$ & $-0.0032(8)$ & $0.0060(8)$ \\
O17 & $0.0250(8)$ & $0.0209(7)$ & $0.0224(8)$ & $-0.0129(6)$ & $-0.0093(6)$ & $0.0028(6)$ \\
O18 & $0.0222(7)$ & $0.0168(7)$ & $0.0346(9)$ & $-0.0061(6)$ & $-0.0164(6)$ & $0.0071(6)$ \\
C19 & $0.0287(12)$ & $0.0290(12)$ & $0.0417(14)$ & $-0.0095(10)$ & $-0.0229(11)$ & $0.0157(10)$ \\
& & & & & & \\
\hline
\end{tabular}

Geometric parameters $\left(\AA,{ }^{\circ}\right)$

\begin{tabular}{|c|c|c|c|}
\hline $\mathrm{C} 1-\mathrm{O} 5$ & $1.474(2)$ & $\mathrm{C} 9-\mathrm{C} 10$ & $1.518(2)$ \\
\hline $\mathrm{C} 1-\mathrm{C} 3$ & $1.508(3)$ & C9-H9A & 0.9700 \\
\hline $\mathrm{C} 1-\mathrm{C} 2$ & $1.517(3)$ & $\mathrm{C} 9-\mathrm{H} 9 \mathrm{~B}$ & 0.9700 \\
\hline $\mathrm{C} 1-\mathrm{C} 4$ & $1.521(3)$ & $\mathrm{C} 10-\mathrm{O} 11$ & $1.220(2)$ \\
\hline $\mathrm{C} 2-\mathrm{H} 2 \mathrm{~A}$ & 0.9600 & $\mathrm{C} 10-\mathrm{N} 12$ & $1.366(2)$ \\
\hline $\mathrm{C} 2-\mathrm{H} 2 \mathrm{~B}$ & 0.9600 & $\mathrm{~N} 12-\mathrm{C} 13$ & $1.406(2)$ \\
\hline $\mathrm{C} 2-\mathrm{H} 2 \mathrm{C}$ & 0.9600 & $\mathrm{~N} 12-\mathrm{H} 12 \mathrm{~A}$ & 0.8600 \\
\hline $\mathrm{C} 3-\mathrm{H} 3 \mathrm{~A}$ & 0.9600 & $\mathrm{C} 13-\mathrm{C} 14$ & $1.335(3)$ \\
\hline $\mathrm{C} 3-\mathrm{H} 3 \mathrm{~B}$ & 0.9600 & $\mathrm{C} 13-\mathrm{C} 16$ & $1.494(3)$ \\
\hline $\mathrm{C} 3-\mathrm{H} 3 \mathrm{C}$ & 0.9600 & $\mathrm{C} 14-\mathrm{Br} 15$ & $1.8715(19)$ \\
\hline $\mathrm{C} 4-\mathrm{H} 4 \mathrm{~A}$ & 0.9600 & $\mathrm{C} 14-\mathrm{H} 14 \mathrm{~A}$ & 0.9300 \\
\hline $\mathrm{C} 4-\mathrm{H} 4 \mathrm{~B}$ & 0.9600 & $\mathrm{C} 16-\mathrm{O} 17$ & $1.204(2)$ \\
\hline $\mathrm{C} 4-\mathrm{H} 4 \mathrm{C}$ & 0.9600 & $\mathrm{C} 16-\mathrm{O} 18$ & $1.337(2)$ \\
\hline $\mathrm{O} 5-\mathrm{C} 6$ & $1.345(2)$ & $\mathrm{O} 18-\mathrm{C} 19$ & $1.447(2)$ \\
\hline $\mathrm{C} 6-\mathrm{O} 7$ & $1.229(2)$ & C19-H19A & 0.9600 \\
\hline $\mathrm{C} 6-\mathrm{N} 8$ & $1.338(2)$ & C19-H19B & 0.9600 \\
\hline $\mathrm{N} 8-\mathrm{C} 9$ & $1.446(2)$ & $\mathrm{C} 19-\mathrm{H} 19 \mathrm{C}$ & 0.9600 \\
\hline $\mathrm{N} 8-\mathrm{H} 8 \mathrm{~A}$ & 0.8600 & & \\
\hline $\mathrm{O} 5-\mathrm{C} 1-\mathrm{C} 3$ & $110.80(16)$ & $\mathrm{C} 9-\mathrm{N} 8-\mathrm{H} 8 \mathrm{~A}$ & 119.5 \\
\hline $\mathrm{O} 5-\mathrm{C} 1-\mathrm{C} 2$ & $109.75(16)$ & $\mathrm{N} 8-\mathrm{C} 9-\mathrm{C} 10$ & $111.87(15)$ \\
\hline $\mathrm{C} 3-\mathrm{C} 1-\mathrm{C} 2$ & $112.96(18)$ & $\mathrm{N} 8-\mathrm{C} 9-\mathrm{H} 9 \mathrm{~A}$ & 109.2 \\
\hline $\mathrm{O} 5-\mathrm{C} 1-\mathrm{C} 4$ & $101.56(15)$ & $\mathrm{C} 10-\mathrm{C} 9-\mathrm{H} 9 \mathrm{~A}$ & 109.2 \\
\hline
\end{tabular}




\begin{tabular}{|c|c|}
\hline $\mathrm{C} 3-\mathrm{C} 1-\mathrm{C} 4$ & $110.90(18)$ \\
\hline $\mathrm{C} 2-\mathrm{C} 1-\mathrm{C} 4$ & $110.29(18)$ \\
\hline $\mathrm{C} 1-\mathrm{C} 2-\mathrm{H} 2 \mathrm{~A}$ & 109.5 \\
\hline $\mathrm{C} 1-\mathrm{C} 2-\mathrm{H} 2 \mathrm{~B}$ & 109.5 \\
\hline $\mathrm{H} 2 \mathrm{~A}-\mathrm{C} 2-\mathrm{H} 2 \mathrm{~B}$ & 109.5 \\
\hline $\mathrm{C} 1-\mathrm{C} 2-\mathrm{H} 2 \mathrm{C}$ & 109.5 \\
\hline $\mathrm{H} 2 \mathrm{~A}-\mathrm{C} 2-\mathrm{H} 2 \mathrm{C}$ & 109.5 \\
\hline $\mathrm{H} 2 \mathrm{~B}-\mathrm{C} 2-\mathrm{H} 2 \mathrm{C}$ & 109.5 \\
\hline $\mathrm{C} 1-\mathrm{C} 3-\mathrm{H} 3 \mathrm{~A}$ & 109.5 \\
\hline $\mathrm{C} 1-\mathrm{C} 3-\mathrm{H} 3 \mathrm{~B}$ & 109.5 \\
\hline $\mathrm{H} 3 \mathrm{~A}-\mathrm{C} 3-\mathrm{H} 3 \mathrm{~B}$ & 109.5 \\
\hline $\mathrm{C} 1-\mathrm{C} 3-\mathrm{H} 3 \mathrm{C}$ & 109.5 \\
\hline $\mathrm{H} 3 \mathrm{~A}-\mathrm{C} 3-\mathrm{H} 3 \mathrm{C}$ & 109.5 \\
\hline $\mathrm{H} 3 \mathrm{~B}-\mathrm{C} 3-\mathrm{H} 3 \mathrm{C}$ & 109.5 \\
\hline $\mathrm{C} 1-\mathrm{C} 4-\mathrm{H} 4 \mathrm{~A}$ & 109.5 \\
\hline $\mathrm{C} 1-\mathrm{C} 4-\mathrm{H} 4 \mathrm{~B}$ & 109.5 \\
\hline $\mathrm{H} 4 \mathrm{~A}-\mathrm{C} 4-\mathrm{H} 4 \mathrm{~B}$ & 109.5 \\
\hline $\mathrm{C} 1-\mathrm{C} 4-\mathrm{H} 4 \mathrm{C}$ & 109.5 \\
\hline $\mathrm{H} 4 \mathrm{~A}-\mathrm{C} 4-\mathrm{H} 4 \mathrm{C}$ & 109.5 \\
\hline $\mathrm{H} 4 \mathrm{~B}-\mathrm{C} 4-\mathrm{H} 4 \mathrm{C}$ & 109.5 \\
\hline $\mathrm{C} 6-\mathrm{O} 5-\mathrm{C} 1$ & $121.71(14)$ \\
\hline $\mathrm{O} 7-\mathrm{C} 6-\mathrm{N} 8$ & $124.52(17)$ \\
\hline $\mathrm{O} 7-\mathrm{C} 6-\mathrm{O} 5$ & $125.30(17)$ \\
\hline $\mathrm{N} 8-\mathrm{C} 6-\mathrm{O} 5$ & $110.18(16)$ \\
\hline $\mathrm{C} 6-\mathrm{N} 8-\mathrm{C} 9$ & $120.96(15)$ \\
\hline $\mathrm{C} 6-\mathrm{N} 8-\mathrm{H} 8 \mathrm{~A}$ & 119.5 \\
\hline $\mathrm{C} 3-\mathrm{C} 1-\mathrm{O} 5-\mathrm{C} 6$ & $62.8(2)$ \\
\hline $\mathrm{C} 2-\mathrm{C} 1-\mathrm{O} 5-\mathrm{C} 6$ & $-62.6(2)$ \\
\hline $\mathrm{C} 4-\mathrm{C} 1-\mathrm{O} 5-\mathrm{C} 6$ & $-179.35(17)$ \\
\hline $\mathrm{C} 1-\mathrm{O} 5-\mathrm{C} 6-\mathrm{O} 7$ & $-4.7(3)$ \\
\hline $\mathrm{C} 1-\mathrm{O} 5-\mathrm{C} 6-\mathrm{N} 8$ & $174.87(15)$ \\
\hline $\mathrm{O} 7-\mathrm{C} 6-\mathrm{N} 8-\mathrm{C} 9$ & $-4.3(3)$ \\
\hline $\mathrm{O} 5-\mathrm{C} 6-\mathrm{N} 8-\mathrm{C} 9$ & $176.12(15)$ \\
\hline $\mathrm{C} 6-\mathrm{N} 8-\mathrm{C} 9-\mathrm{C} 10$ & $-86.1(2)$ \\
\hline $\mathrm{N} 8-\mathrm{C} 9-\mathrm{C} 10-\mathrm{O} 11$ & $-38.0(3)$ \\
\hline $\mathrm{N} 8-\mathrm{C} 9-\mathrm{C} 10-\mathrm{N} 12$ & $143.70(16)$ \\
\hline $\mathrm{O} 11-\mathrm{C} 10-\mathrm{N} 12-\mathrm{C} 13$ & $-2.5(3)$ \\
\hline
\end{tabular}

N8-C9-H9B

$\mathrm{C} 10-\mathrm{C} 9-\mathrm{H} 9 \mathrm{~B}$

H9A-C9-H9B

$\mathrm{O} 11-\mathrm{C} 10-\mathrm{N} 12$

$\mathrm{O} 11-\mathrm{C} 10-\mathrm{C} 9$

$\mathrm{N} 12-\mathrm{C} 10-\mathrm{C} 9$

C10-N12-C13

$\mathrm{C} 10-\mathrm{N} 12-\mathrm{H} 12 \mathrm{~A}$

C13-N12-H12A

C14-C13-N12

$\mathrm{C} 14-\mathrm{C} 13-\mathrm{C} 16$

N12-C13-C16

$\mathrm{C} 13-\mathrm{C} 14-\mathrm{Br} 15$

C13-C14-H14A

$\mathrm{Br} 15-\mathrm{C} 14-\mathrm{H} 14 \mathrm{~A}$

O17-C16-O18

$\mathrm{O} 17-\mathrm{C} 16-\mathrm{C} 13$

$\mathrm{O} 18-\mathrm{C} 16-\mathrm{C} 13$

C16-O18-C19

O18-C19-H19A

O18-C19-H19B

H19A-C19-H19B

O18-C19-H19C

H19A-C19-H19C

H19B-C19-H19C

C9- $110-\mathrm{N} 12-\mathrm{C} 13$

$\mathrm{C} 10-\mathrm{N} 12-\mathrm{C} 13-\mathrm{C} 14$

$\mathrm{C} 10-\mathrm{N} 12-\mathrm{C} 13-\mathrm{C} 16$

$\mathrm{N} 12-\mathrm{C} 13-\mathrm{C} 14-\mathrm{Br} 15$

$\mathrm{C} 16-\mathrm{C} 13-\mathrm{C} 14-\mathrm{Br} 15$

$\mathrm{C} 14-\mathrm{C} 13-\mathrm{C} 16-\mathrm{O} 17$

$\mathrm{N} 12-\mathrm{C} 13-\mathrm{C} 16-\mathrm{O} 17$

$\mathrm{C} 14-\mathrm{C} 13-\mathrm{C} 16-\mathrm{O} 18$

$\mathrm{N} 12-\mathrm{C} 13-\mathrm{C} 16-\mathrm{O} 18$

$\mathrm{O} 17-\mathrm{C} 16-\mathrm{O} 18-\mathrm{C} 19$

C13-C16-O18-C19
109.2

109.2

107.9

$122.87(17)$

$122.69(16)$

$114.41(16)$

$121.43(16)$

119.3

119.3

124.27 (18)

118.50 (17)

$116.92(16)$

$123.17(15)$

118.4

118.4

$124.26(18)$

124.08 (18)

111.61 (16)

115.57 (16)

109.5

109.5

109.5

109.5

109.5

109.5

$175.79(16)$

130.6 (2)

$-55.9(2)$

-0.9 (3)

$-174.28(13)$

$159.29(19)$

-14.6 (3)

-18.4 (2)

$167.72(16)$

$-4.6(3)$

173.12 (17)

Hydrogen-bond geometry $\left(\AA,{ }^{\circ}\right)$

\begin{tabular}{lllll}
\hline$D-\mathrm{H} \cdots A$ & $D-\mathrm{H}$ & $\mathrm{H} \cdots A$ & $D \cdots A$ & $D-\mathrm{H} \cdots A$ \\
\hline $\mathrm{C} 2-\mathrm{H} 2 A \cdots \mathrm{O} 7$ & 0.96 & 2.51 & $3.058(2)$ & 116 \\
$\mathrm{C} 3-\mathrm{H} 3 A \cdots \mathrm{O} 7$ & 0.96 & 2.44 & $3.007(3)$ & 117 \\
$\mathrm{~N} 8-\mathrm{H} 8 A \cdots \mathrm{O} 17^{\mathrm{i}}$ & 0.86 & 2.19 & $3.018(2)$ & 162 \\
$\mathrm{C} 9-\mathrm{H} 9 A \cdots \mathrm{O} 11^{\mathrm{i}}$ & 0.97 & 2.61 & $3.255(2)$ & 124 \\
$\mathrm{~N} 12-\mathrm{H} 12 A \cdots \mathrm{O} 7^{\mathrm{ii}}$ & 0.86 & 2.04 & $2.901(2)$ & 174
\end{tabular}


supporting information

$\mathrm{C} 14-\mathrm{H} 14 A \cdots \mathrm{O} 11^{\mathrm{iii}}$

$\mathrm{C} 19-\mathrm{H} 19 B^{\cdots} \cdots \mathrm{Br} 15^{\mathrm{iii}}$

Symmetry codes: (i) $-x+2,-y,-z+1$; (ii) $-x+3,-y,-z+1$; (iii) $-x+2,-y+1,-z+1$.
2.43

3.14
3.095 (2)

$3.668(3)$
129

117 